\title{
Case predictive and prognostic Biomarkers for Schizophrenia
}

Dr Stephanie Fryar-Williams ${ }^{1}$ Youth in Mind Research Institute, Adelaide, SA, Australia. ${ }^{2}$ The Queen Elizabeth Hospital, Woodville, SA, Australia, Basil Hetzel Institute for Translational Health Research, Woodville, SA.

Dr Graeme Tucker ${ }^{4}$ University of Adelaide, Adelaide, South Australia.

Dr Peter Clements 4 University of Adelaide, Waite Research Institute, South Australia.

Dr Yichao Huang ${ }^{4}$ University of Adelaide, Waite Research Institute, South Australia.

Dr Jörg Strobel ${ }^{4}$ The University of Adelaide, Adelaide, South Australia.

\section{Objectives}

The Mental Health Biomarker Project (2010-2019) sought to

- discover biomarkers for schizophrenia related to the main rate-limiting MTHFR C677T gene for methylation.

- test the predictive capacity of these biomarkers to enhance traditional symptom-based assessments.

- integrate these markers with sensory processing markers to allow more comprehensive understanding of psychosis mechanics and prognosis of outcomes.

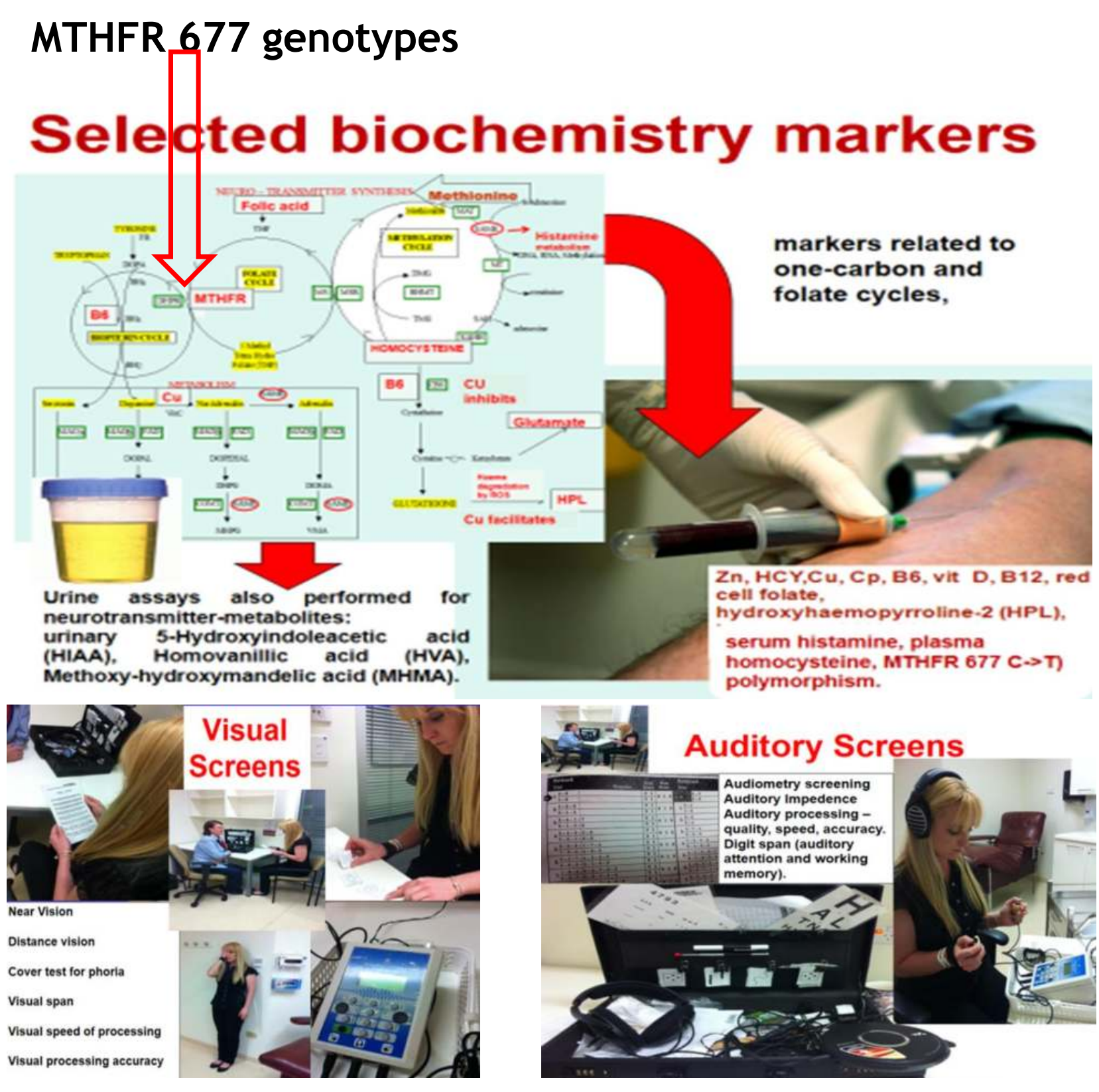

The biomarkers also explain:

- Heterogeneity in schizophrenia.

- The importance of genotype differentiation for obtaining meaningful research results in Psychiatry

Limitations: Pooled data from case-control retrospective study. Selectively medicated patient-sample.

Marginal prevalence for MTHFR 677 TT sample.

\section{Materials and Methods}

67 well characterised, DSM IV-R-diagnosed, multi-ethnic, non-acute cases aged 18-60 years from ward and community settings and 67 normal subjects had blood and urine testing for intermediate peptides and cofactors in biochemistry pathways associated the strategically functioning MTHFR C677T gene. Functional outcomes measured by Symptom intensity index (SIR) on Brief Psychiatric Rating Scale, Global Assessment of Function (GAF), Clinical Global Impression (CGI), Social and occupational function scale (SOFAS) Duration of Illness (DOI) hospitalization frequency (Hf) and Disability Support (DS). Case control data was paired for age and sex after exclusion of ineligible participants. Data analysis was by internally validated receiver operating characteristics (ROC curves), Spearman's correlates, logistic and linear regression with likelihood ratio (LR), odds ratio (OR), R2 and Chi2 probability for $95 \% \mathrm{Cl}$. Inclusion criteria: $18-60$ years, DSM IV-R-diagnosed and verified by DSM IV-R checklist. Schizophrenia or schizo-affective disorder. Stable medication, Exclusion criteria: Clozapine, Olanzapine, anti-histamines or vitamin therapy. Substance abuse, upper-respiratory-tract infections, intellectual, visual or auditory disability, clinical history of head-injury, disorientation or extrapyramidal signs.

\section{Results}

- Methylation cofactors and their related catecholamine and oxidative stress levels achieved biomarker status and differentiated between MTHFR 677 genotypes.

- Genotype-dependent classification models achieved $90-95 \% \%$ sensitivity and specificity for schizophrenia diagnosis, based upon blood and urine results, alone.

- Taken together, biomedical and sensory processing variables predicted diagnosis for MTHFR 677 genotypes with between 92 and $100 \%$ sensitivity and specificity.

- The biomarkers predict severity, disability and costcare burden for persons with schizophrenia.

- If validated in wider populations, the biomarkers carry potential for genotype guided treatment by algorithmic prediction and enzyme cofactor adjustments.

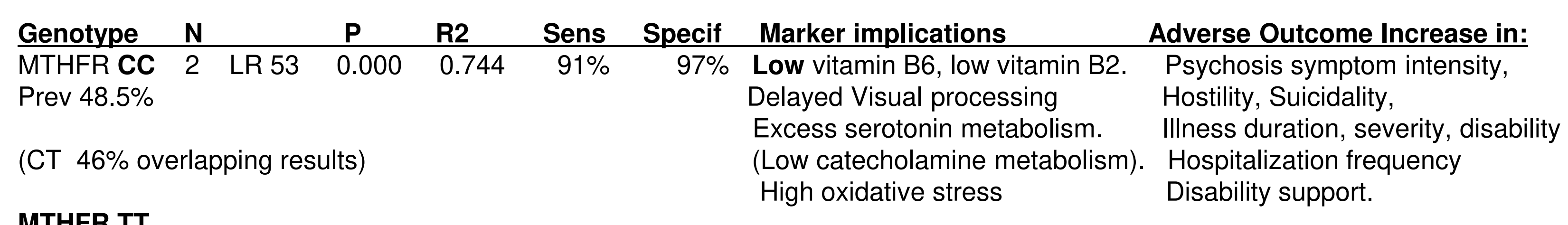

MTHFR TT

Prev 5.2\%

$7 \quad$ OR $2.2 \quad 0.000$

$100 \%$

100\% Elevated vitamin B2/creatinine (Low zinc, high catecholamine metabolism correlates)

(Preserved sensory processing) 